\title{
Taking a Breath of the Wild: are geoscientists more effective than non-geoscientists in determining whether video game world landscapes are realistic?
}

\author{
Rolf Hut $^{1}$, Casper Albers ${ }^{2}$, Sam Illingworth ${ }^{3}$, and Chris Skinner ${ }^{4}$ \\ ${ }^{1}$ Water Resources Engineering, Faculty of Civil Engineering and Geosciences, Delft University of Technology, \\ Delft, the Netherlands \\ ${ }^{2}$ Heymans Institute for Psychological Research, University of Groningen, Groningen, the Netherlands \\ ${ }^{3}$ Department of Natural Sciences, Manchester Metropolitan University, Manchester, UK \\ ${ }^{4}$ Energy and Environment Institute, University of Hull, Hull, UK
}

Correspondence: Rolf Hut (r.w.hut@tudelft.nl)

Received: 11 March 2019 - Discussion started: 15 March 2019

Revised: 12 July 2019 - Accepted: 5 August 2019 - Published: 13 August 2019

Abstract. From the wilderness of Hyrule, the continent of Tamriel, and the geographies of Middle Earth, players of video games are exposed to wondrous, fantastic, but ultimately fake, landscapes. Given the time people may spend in these worlds compared to the time they spend being trained in geoscience, we wondered whether expert geoscientists would differ from non-geoscientists in whether they judge the landscapes in these video games to be "realistic". Since video games present a great opportunity for tangential learning, it would be a missed opportunity if it turns out that features obviously fake to geoscientists are perceived as plausible by non-geoscientists.

To satisfy our curiosity and answer this question, we conducted a survey where we asked people to judge both photos from real landscapes as well as screenshots from the recent The Legend of Zelda: Breath of the Wild video game on how likely they thought the features in the picture were to exist in the real world. Since game world screenshots are easily identified based on their rendered, pixelated nature, we preprocessed all pictures with an artistic "Van Gogh" filter that removed the rendered nature but retained the dominant landscape features.

We found that there is a small but significant difference between geoscientists and non-geoscientists, with geoscientists being slightly better at judging which pictures are from the real world versus from the video game world. While significant, the effect is small enough to conclude that fantastical worlds in video games can be used for tangential learning on geoscientific subjects.

\section{Introduction}

Modern video games often provide players with fictional worlds that the players (characters) can explore. While some game worlds include utterly alien (Star Wars: The Old Republic, Horizon Zero Dawn) or dense urban landscapes (Grand Theft Auto 5, Spiderman), many offer a world that has large stretches of "natural environment" as could be found on Earth (The Legend of Zelda: Breath of the Wild, Middle-earth: Shadow of Mordor, Red Dead Redemption). However, many of these natural environments contain elements that are, from a geoscientific point of view, unrealistic. This could be due either to the restraints of having to provide an engaging video game or to the game designers wanting to present the players with a fantastic, epic setting for their video game. The most strikingly unrealistic aspect of many video games is that different climate zones are often represented on a relatively small area. For example, the entire world of Red Dead Redemption 2, although considered massive for a game, only covers $75 \mathrm{~km}^{2}$ (Reddit, 2018), yet includes deserts, prairies, grassy planes, forests, and mountain ranges. Similarly, the world of Hyrule in The Legend of Zelda: Breath of the Wild was designed to be "about as big as Kyoto" (Webster, 2017), yet it includes, again, sweltering 
sand deserts, mountain ranges, swamps, and a freezing arctic tundra.

Video games have a great potential for tangential learning, i.e. learning things about the real world as a tangential benefit while primarily enjoying the experience (Portnow, 2012; Mozelius et al., 2017). The tangential learning opportunities of video games have been studied elsewhere (see e.g. Breuer and Bente, 2010; Mozelius et al., 2017); however, what has not yet been fully addressed is the extent to which this tangential learning could lead to misinformation if the game world was presented in a manner that was incongruent with reality. As such, we wondered whether presenting unrealistic geo-features in a video game might lead to erroneous learning; i.e. might gamers pick up flawed knowledge of geo-features in our real world because they are presented as realistic within the game world? To test this hypothesis, we conducted a survey in which people were presented with images from the real world and screenshots from a video game before being asked to rate how realistic they thought the depicted landscape was. To make sure that the different images were not recognizable as "from a video game" versus "from the real world" (e.g. due to rendering and pixelation), while still depicting the landscapes we wanted to study, we used an automated artistic "van Gogh" filter available at LunaPic.com (LunaPic, 2015). This filter hides the detailed nature of the image by replacing pixels with brush strokes, but retains the overall shape of geological features depicted in the image.

Video games are often reported in the popular press as having supposed negative consequences, such as those associated with addiction, violence, and isolation (Ferguson, 2007). However, several studies (dating back to the 1980s) have also shown that there are many positive benefits to be gained from playing video games, such as improving the hand-to-eye coordination, self-esteem, and even social interactions of the players (see e.g. Griffiths, 2002; Granic et al., 2014; Wang et al., 2018). The educational benefits of playing video games have also been well studied and documented (Squire, 2002, 2003; Gee, 2003; Mayer, 2019), and the potential for video games to contribute towards scientific education is highlighted in the following quote from Gee (2003, p. 20), who states that

Designers face and largely solve an intriguing educational dilemma, one also faced by schools and workplaces: how to get people, often young people, to learn and master something that is long and challenging - and enjoy it, to boot.

As noted by Mayo (2009), video games have tremendous mass appeal, reaching audiences in the hundreds of thousands to millions, and so video games would seem to be an ideal medium through which to communicate geoscientific topics, especially in informal learning environments.

As noted by Dudo et al. (2014), video games now represent one of the primary platforms through which the gen- eral public, and in particular children and adolescents, observe and interact with scientists, and given their global reach and popularity they are fast becoming a key science touch point for non-scientists. As well as being an important tool in engaging non-traditional audiences (Newman et al., 2012), video games offer the opportunity to spark meaningful and organic engagement around a particular topic (Curtis, 2014). However, if video games convey information that is incorrect or misleading, then it might be that this engagement serves to detract from, rather than contribute towards, the development of meaningful scientific discourse by members of the general public (Squire, 2003). In understanding and constructing meaning from video games, individuals process the images and elements of design (Rodríguez Estrada and Davis, 2015), and it is the purpose of this study to better understand how this processing enables non-geoscientific audiences to differentiate between realistic and unrealistic geo-features.

In the methods section below we explain the setup of our survey and the statistical methods used to analyse the results. In the results section we present our findings and, in the conclusions, we contextualize these findings and discuss further opportunities for research. Finally, Appendix A contains a post hoc analysis of the survey data, to look for further interesting patterns. The entire survey is provided in the Supplement.

\section{Methods}

The main question this research seeks to answer is "do people without a background in the geosciences perceive landscapes from game worlds as more realistic compared to those with a background in the geosciences?" To study this question, we took six images from the video game The Legend of Zelda: Breath of the Wild (BotW). The images were chosen to represent a wide variety of landscapes (a volcano, a tropical forest, a grassy plane, etc.) and needed to include geological features as central criteria. The images were chosen such that no clear landmarks that identified it as a fantasy game, such as iconic temples, towers, or Hyrule Castle, were visible in the picture. To select the images used in this study, we constructed a list of landscape types (volcano, arctic, desert, plains, swamps, jungle) that we wished to include in the survey. Author Hut (who has an intimate knowledge of the game world) selected six locations that did not include any recognizable features and made screenshots using the in-game camera option. Each image was used as input in a reversed image search in the Google search engine. From the search result real world images were handpicked. To determine whether participants could distinguish made-up landscapes in video games from real landscapes, for each picture taken from Bot $W$ an accompanying image from the real world was sought by doing a reverse image search. Since the BotW screenshots can be easily recognized due to their rendered nature, we choose to use an automated artistic 


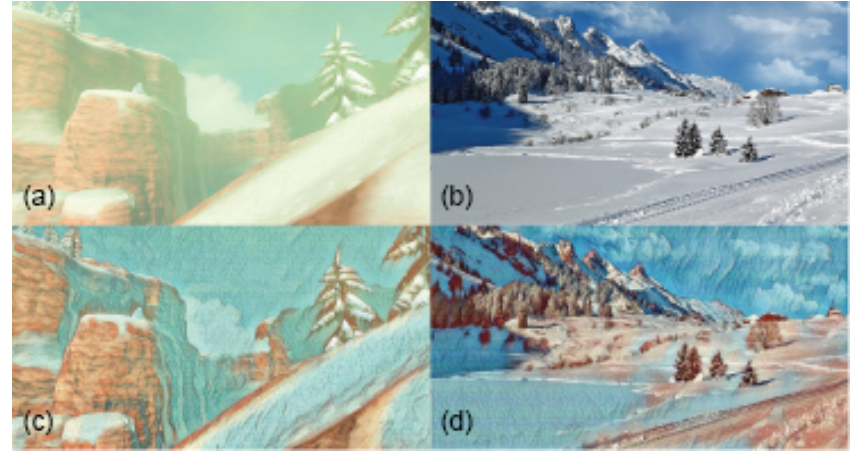

Figure 1. Two images used in the survey. Panels (a) and (b) are original and (c) and (d) are processed through the "van Gogh" filter. The left two images are from the video game The Legend of Zelda: Breath of the Wild and the right two images are from the real world. The bottom two figures were presented in the survey with the question "Knowing that this picture has gone through a "van Gogh"-filter, how likely do you think it is that the features depicted in the artistic renderings could exist in the real world?". We used a 10 -point scale where $1=$ completely unlikely to $10=$ completely likely. High-resolution versions of images (originals and after filters were applied) are available in the Supplement.

filter on both the BotW screenshots and on the real world images. The filter needed to alter both types of images enough to not be able to distinguish them based on the rendering nature of the screenshots yet retain the essential geo-features of the landscape. To make sure that all of the images were treated equally, we needed a filter that works algorithmically and does not require input from a human. Many such filters exist online, yet most of them either failed in removing the rendered nature of the video game images or else modified the image too much, removing any of the geoscientific content. The "van Gogh" filter (available through LunaPic, 2015) was chosen as it was one of the few filters which retained the geological features of the image while hiding the rendered nature of the video game images. Figure 1 shows how two images (a Bot $W$ screenshot in panel a and a real world photo in panel b) were transformed using the filter. Both the original as well as the filtered figures are available in the Supplement.

\section{Survey design}

To test our hypothesis, we wanted to know whether people are capable of recognizing fake landscapes from video games and whether geoscientists are better at this than non-geoscientists. When creating an assessment on whether someone thinks a certain photo is real or faked, there are basically two options to do so. One option is to ask "Do you think this photo is real?" via a yes/no question; the other is to ask "How certain are you that this photo is real?" on an ordinal scale. The benefit of such ordinal scales is that they can incorporate more nuance than a simple dichotomy, as one can also indicate that one is not sure. Such Likert scales are extremely common, especially within the social sciences. Common scales are five-, seven-, and nine-point scales. See Lord and Novick (2008) or Kaplan and Saccuzzo (2017) for more background on questionnaire design. Therefore, for all the pictures generated as described above, we asked the question "Knowing that this picture has gone through a "van Gogh"filter, how likely do you think it is that the features depicted in the artistic renderings could exist in the real world?". We used a 10-point scale where $1=$ completely unlikely to $10=$ completely likely.

To distinguish between people with and without a background in the geosciences, we asked the question "Do you consider yourself a geoscientist?".

We wanted to exclude participants who had previously played $B o t W$, as they could potentially recognize places from the video game, skewing the results. At the same time, we did not want to alert everyone to the fact that the pictures they were looking at were taken from this particular video game; therefore, we added a broad question asking participants which video games they had played in the last year, which included BotW.

As additional background information to be able to do post hoc analyses, we added questions on age, gender, and highest completed education level. The entire survey, including the required legal statements on voluntary participation, proper handling of private information, and the option to quit at any time, is provided in the Supplement.

The survey was carried out using two methods: printed versions of the survey were handed out at the European Geoscience Union (EGU) General Assembly 2018 in Vienna. In this way we intended to reach people with a background in the geosciences. After the assembly, an online version of the survey, designed in Google Forms and available through http://rolfhut.nl/botw (Hut, 2018), was announced using the social media accounts of the authors during the conference and in the week afterwards ( 8 through 20 April 2018). Advertising a survey through social media runs the risk of selecting survey participants from the biased social circle of the authors. However, Côté and Darling (2018) showed that above 1000 followers, a more diverse public is reached through Twitter. Given that all the authors shared the survey through their personal Twitter accounts, and that each of these accounts has more than 2000 followers, we are confident that an acceptably diverse public was reached using this approach.

\section{Statistical analyses}

All 12 pictures, 6 Bot $W$ pictures and 6 real pictures, were rated by all the participants on a 1-10 scale. The rating on photo $i$ by participant $j$ is denoted by $r_{i, j}$. As a first step, we studied on a picture-by-picture basis whether the mean ratings of geoscientists differ from those of non-geoscientists. 
For this, we used a Student's $t$ test with Bonferroni correction to account for multiple testing.

However, our main interest was not in the individual pictures, but in the overall message from the 12 pictures. An overall penalty score per person was therefore constructed. For each photo, participants rated the photos on a scale from 1 (= fake) to 10 (= real). The best answer they can give is 1 for the six game world photos and 10 for the six real world ones. For each photo, the distance between the given answer and the best answer was calculated (thus 0 when the participant is fully correct up to 9 when they are fully wrong). The absolute values of the 12 distance scores for the photos were added, and the resulting metric represents a penalty, as a low score is good (the perfect candidate scores 0 , and one who's as wrong as it gets scores $12 \cdot 9=108)$.

To operationalize this we defined $K=(10,1,1,10,10,1$, $1,10,10,1,1,10)$ as the vector of ideal answers ("1" for each picture from BotW and "10" for the real ones). We then computed the score for each person in the survey by counting the sum of the distances to this optimal answer:

Penalty $_{i}=\sum_{j=1}^{12}\left|r_{i, j}-K_{i}\right|$.

The score patterns of geoscientists and non-geoscientists will be compared both visually and by using a Student's $t$ test.

All hypothesis tests were carried out two-sided, and the code to run these analyses as well as the anonymized survey files are available at Albers and Hut (2019).

\section{Results}

A total of 163 people completed the survey. Four participants did not score all pictures and were excluded from the data. Furthermore, 17 participants indicated that they had played $B o t W$ and were also excluded. Of the remaining 142 participants, $84(59 \%)$ of them indicated that they were a geoscientist. The average rating and standard deviation of the ratings for geoscientists and non-geoscientists are provided in Table 1. Full aggregated scores per picture per group (geoscientist versus non-geoscientist) are provided in the Appendix (Tables A1 and A2). Figure 2 shows the distribution of penalty scores for both groups. From the visual comparison, we observe that geoscientists, on average, have lower penalty scores than non-geoscientists. Also from the table we see that compared to the non-geoscientists the geoscientists gave higher scores for the real world pictures and lower scores for the in-game screenshots, indicating that they are better at telling the difference. Since our research only tests whether people recognize video game world images as not realistic, we can not say through what mechanism geoscientists arrive at their better score compared to nongeoscientists. We hypothesize that this could be an effect of their training or an effect of being exposed to many real geoscientific images during their career. Concluding that provid-
Table 1. Average rating and SD per picture for geoscientists and non-geoscientists. The first six rows concern real pictures, the last six Bot W pictures.

\begin{tabular}{lrr|lr}
\hline & \multicolumn{2}{c}{ Geoscientists } & \multicolumn{2}{c}{ Non-geoscientists } \\
\cline { 2 - 5 } & Mean & SD & Mean & SD \\
\hline Picture 1 & 7.952 & 2.409 & 7.655 & 2.453 \\
Picture 4 & 9.476 & 0.814 & 8.879 & 1.836 \\
Picture 5 & 9.071 & 1.438 & 8.690 & 1.749 \\
Picture 8 & 8.119 & 2.515 & 7.776 & 2.435 \\
Picture 9 & 9.131 & 1.387 & 8.638 & 1.861 \\
Picture 12 & 7.726 & 2.481 & 7.603 & 2.554 \\
\hline Real pictures & 8.579 & 1.112 & 8.207 & 1.164 \\
\hline Picture 2 & 3.702 & 2.104 & 5.017 & 2.544 \\
Picture 3 & 6.274 & 2.500 & 6.621 & 2.553 \\
Picture 6 & 6.381 & 2.737 & 6.086 & 2.529 \\
Picture 7 & 5.798 & 2.419 & 5.948 & 2.228 \\
Picture 10 & 5.940 & 2.485 & 6.655 & 2.482 \\
Picture 11 & 6.381 & 2.755 & 6.224 & 2.596 \\
\hline BotW pictures & 5.746 & 1.685 & 6.092 & 1.566 \\
\hline
\end{tabular}

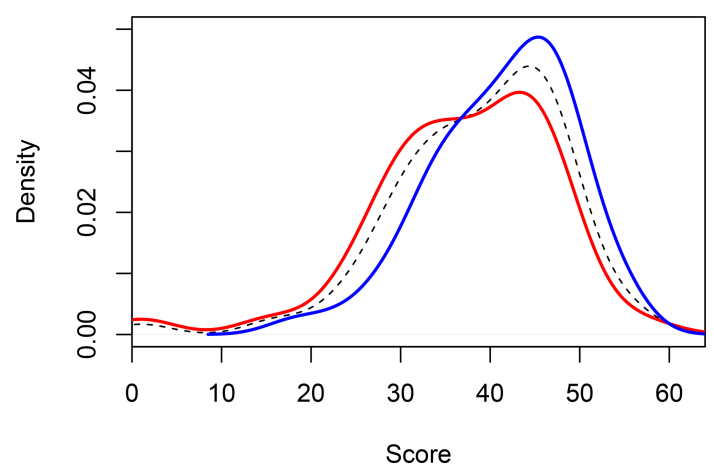

Figure 2. Distribution of the penalty scores. The red curve denotes the geoscientists, the blue one the non-geoscientists. The dashed black curve shows the density for the whole sample.

ing non-geoscientists with either more training or more exposure to geoscientific images, in order to improve their ability to recognize non-realistic landscapes in video games, is not possible based on these results. More qualitatively focused follow-up research could potentially unearth this reason.

On an individual picture level, only the difference for Picture 2 is statistically significant $(t=3.358$, degrees of freedom, $\mathrm{df}=140, p_{\text {Bonf }}=0.012$ ). This hints that the difference between geoscientists and non-geoscientists is most likely small.

Statistically testing our overall hypothesis by combining the information over all 12 pictures, we find that geoscientists indeed score better at separating fake landscapes from real ones $(t=2.704, \mathrm{df}=140, p=0.008)$. However, the effect size is rather small: the mean combined penalty in the non-geoscientist group is 41.31 , and in the geoscientist group 
it is 37.00 . Thus, geoscientists perform about $10 \%$ better, according to the metric in Eq. (1). When including additional covariates (whether or not someone is a gamer, gender, and age) in the analyses, the message remains that geoscientists perform about $10 \%$ better, i.e. even when correcting for those covariates, the difference between geoscientists and non-geoscientists remains significant (see Table A3). Out of the 84 experts, 34 filled in the questionnaire on paper and the rest digitally. Of the 58 non-geoscientists, only 4 filled in the questionnaire on paper. Different modes of administration for both groups could have had a small effect on the outcomes.

Other interesting patterns are visible in the collected data. For example (Table A3), the effects of being a self-identified gamer have no influence on the result. This surprises us, since we would expect that people being exposed more to video games might have a better sense for which landscapes are from a video game. We have to stress that this is a post hoc analyses that only results in a hypothesis that needs further testing.

\section{Conclusions}

We have demonstrated that while geoscientists might be slightly, but statistically significantly, better at separating real world photos of landscapes from video game screenshots, non-geoscientists are still capable of identifying landscapes from a video game, even when both the real world photos and the video game screenshots are filtered through an artistic "van Gogh" filter. This suggests that people recognize the natural features in video game worlds for the fantastical settings that they are. On a 10-point scale non-geoscientists rate real world images on average 2.115 points higher than images from the video game. Geoscientists rate real world images 2.833 points higher. Whether people are able to distinguish factual information (a volcano is hot) from erroneous information (an arctic tundra located within a 5 min walk from a sweltering desert) within the context of a video game is a question that requires further research.

Care needs to be taken when interpreting the results of this study. While we clearly asked people to indicate whether the features in the picture could exist in the real world, and we did not ask them to judge whether the picture is from a video game, there is a chance that some people answered the question with this in mind. To prevent this, we used the "van Gogh" filter to hide the fact that the video game screenshots were from a rendered video game image. However, people with experience in playing video games might still look for tell-tale signs of video game generated images. Landscapes in video games are, of course, designed by artists who draw on the real world for inspiration. Different video games will have different levels of adherence to real world inspirations, depending on the aesthetic sensibilities of the game world or limitations of the software used to create it. Our research focused on this question: can non-geoscientific experts correctly identify non-realistic aspects of landscapes in video games? The paper survey was handed out at the EGU General Assembly 2018 to target geoscientists. However, this was done during the "games in geoscience" session, potentially over-representing gamers among geoscientists. Post hoc analyses showed no significant over-representation of gamers among geoscientists (see Table A3). More detailed qualitative research, guided by the results of this research, could further test the assumptions made in this work and provide insight into the manner by which people determine whether an image is real or not. Finally, the distinction between geoscientists and non-geoscientists is made based on an acknowledgement of formal education in the geosciences. In the non-geoscientist group there is potentially a large difference between people that do, and do not, get exposed to geological features, through hiking, watching documentaries, etc. Follow-up research, either qualitative or quantitative, could include questions on self-perceived level of geoscientific knowledge. Doing so would likely yield further information on how well versed those educated in the geosciences consider themselves to be versus those not educated, perhaps demonstrating a geoscientific version of the Dunning-Kruger effect (Dunning, 2011).

We have shown that even though the difference in the ability to identify whether an image is from a video game or from the real world is significant, the effect size is small. Whilst further study is needed to fully assess the effectiveness of video games when used in this manner, this study indicates that wrongfully interpreting game world landscapes as real is not a risk when aiming to tangentially communicate geoscientific principles.

Code and data availability. The anonymized survey results, as well as the code that was used to perform the statistical analyses, are available through Albers and Hut (2019, https://doi.org/10.17605/ OSF.IO/53VDS). 
Appendix A: Additional tables

Table A1. Frequencies of ratings by geoscientists for all pictures. The first six columns are the real pictures, the last six columns the Bot $W$ pictures.

\begin{tabular}{l|rrrrrr|rrrrrr|r}
\hline & P1 & P4 & P5 & P8 & P9 & P12 & P2 & P3 & P6 & P7 & P10 & P11 & $\sum$ \\
\hline Grade: 1 & 1 & 0 & 1 & 3 & 0 & 3 & 13 & 2 & 2 & 4 & 2 & 3 & 34 \\
Grade: 2 & 1 & 0 & 0 & 3 & 0 & 2 & 12 & 5 & 3 & 2 & 5 & 2 & 35 \\
Grade: 3 & 5 & 0 & 0 & 2 & 0 & 4 & 22 & 8 & 9 & 9 & 7 & 11 & 77 \\
Grade: 4 & 5 & 0 & 0 & 2 & 1 & 1 & 12 & 6 & 12 & 11 & 13 & 13 & 76 \\
Grade: 5 & 3 & 0 & 1 & 1 & 2 & 5 & 9 & 12 & 13 & 18 & 15 & 5 & 84 \\
Grade: 6 & 5 & 0 & 2 & 5 & 3 & 3 & 4 & 8 & 5 & 6 & 7 & 6 & 54 \\
Grade: 7 & 5 & 3 & 4 & 5 & 4 & 11 & 7 & 12 & 6 & 9 & 8 & 9 & 83 \\
Grade: 8 & 12 & 8 & 13 & 13 & 10 & 13 & 4 & 12 & 6 & 11 & 8 & 10 & 120 \\
Grade: 9 & 16 & 19 & 18 & 15 & 13 & 18 & 0 & 11 & 12 & 9 & 13 & 10 & 154 \\
Grade: 10 & 31 & 54 & 45 & 35 & 51 & 24 & 1 & 8 & 16 & 5 & 6 & 15 & 291 \\
\hline
\end{tabular}

Table A2. Frequencies of ratings by non-geoscientists for all pictures. The first six columns are the real pictures, the last six columns the Bot $W$ pictures.

\begin{tabular}{l|rrrrrr|rrrrrr|r}
\hline & P1 & P4 & P5 & P8 & P9 & P12 & P2 & P3 & P6 & P7 & P10 & P11 & $\sum$ \\
\hline Grade: 1 & 1 & 0 & 0 & 1 & 0 & 0 & 5 & 1 & 2 & 0 & 1 & 1 & 12 \\
Grade: 2 & 0 & 0 & 0 & 1 & 2 & 4 & 3 & 2 & 4 & 3 & 2 & 4 & 25 \\
Grade: 3 & 6 & 3 & 1 & 4 & 0 & 0 & 13 & 5 & 5 & 7 & 6 & 6 & 56 \\
Grade: 4 & 4 & 1 & 2 & 3 & 0 & 5 & 6 & 8 & 6 & 8 & 5 & 7 & 55 \\
Grade: 5 & 0 & 0 & 2 & 1 & 2 & 6 & 6 & 5 & 5 & 7 & 4 & 5 & 43 \\
Grade: 6 & 2 & 1 & 1 & 3 & 3 & 2 & 8 & 2 & 7 & 8 & 7 & 7 & 51 \\
Grade: 7 & 5 & 3 & 4 & 5 & 2 & 6 & 7 & 11 & 12 & 7 & 6 & 6 & 74 \\
Grade: 8 & 14 & 7 & 10 & 12 & 13 & 6 & 4 & 7 & 6 & 10 & 10 & 7 & 106 \\
Grade: 9 & 10 & 11 & 11 & 10 & 9 & 9 & 2 & 8 & 5 & 6 & 11 & 9 & 101 \\
Grade: 10 & 16 & 32 & 27 & 18 & 27 & 20 & 4 & 9 & 6 & 2 & 6 & 6 & 173 \\
\hline
\end{tabular}

Table A3. Results of the linear regression model predicting penalty scores from dichotomous variables geoscientist, gamer, and male and continuous variable age. Geoscientists and men score significantly better than non-geoscientists and women, respectively. The effect of gamer and age is non-significant.

\begin{tabular}{lrrrr}
\hline & Estimate & SE & $t$ value & $p$ value \\
\hline (Intercept) & 39.505 & 3.360 & 11.76 & $<0.001$ \\
Geoscientist & -4.125 & 1.653 & -2.50 & 0.014 \\
Gamer & 2.048 & 1.66 & 1.23 & 0.219 \\
Male & -3.499 & 1.63 & -2.15 & 0.034 \\
Age & 0.087 & 0.077 & 1.13 & 0.259 \\
\hline
\end{tabular}


Supplement. The supplement related to this article is available online at: https://doi.org/10.5194/gc-2-117-2019-supplement.

Author contributions. We are using the CASRAI Credit system (Allen et al., 2014) to acknowledge the different roles of the different authors.

- RH was involved in conceptualization, data curation, investigation, methodology, visualization, writing the original draft, and review and editing.

- CA was involved in methodology, writing the original draft (statistical analyses and results), formal analysis, and review and editing.

- SI was involved in conceptualization, writing the original draft (introduction), and review and editing.

- CS was involved in conceptualization and review and editing.

Competing interests. The authors explicitly state that they have no commercial ties to the Nintendo corporation, producer of the Zelda: Breath of the Wild video game. Sam Illingworth is the chief executive editor of Geoscience Communication, but was not involved in the editorial or peer review process of this paper.

Acknowledgements. The authors would like to thank all the people that have participated in the survey.

No funding was received to carry out this research.

Review statement. This paper was edited by Hazel Gibson and reviewed by two anonymous referees.

\section{References}

Albers, C. J. and Hut, R.: Taking a Breath of the Wild, analyses code and data, https://doi.org/10.17605/OSF.IO/53VDS, 2019.

Allen, L., Scott, J., Brand, A., Hlava, M., and Altman, M.: Publishing: Credit Where Credit Is Due, Nature News, 508, 312, https://doi.org/10.1038/508312a, 2014.

Breuer, J. and Bente, G.: Why so serious? On the relation of serious games and learning, Journal for Computer Game Culture, 4, 724, 2010.

Côté, I. M. and Darling, E. S.: Scientists on Twitter: Preaching to the choir or singing from the rooftops?, Facets, 3, 682-694, 2018.

Curtis, V.: Public Engagement Through the Development of Science-Based Computer Games: The Wellcome Trust's "Gamify Your PhD” Initiative, Sci. Commun., 36, 379-387, https://doi.org/10.1177/1075547013492436, 2014.

Dudo, A., Cicchirillo, V., Atkinson, L., and Marx, S.: Portrayals of Technoscience in Video Games: A Potential Avenue for Informal Science Learning, Sci. Commun., 36, 219-247, https://doi.org/10.1177/1075547013520240, 2014.
Dunning, D.: The Dunning-Kruger effect: On being ignorant of one's own ignorance, in: Advances in experimental social psychology, Vol. 44, 247-296, Academic Press, 2011.

Ferguson, C. J.: The Good, The Bad and the Ugly: A Meta-Analytic Review of Positive and Negative Effects of Violent Video Games, Psychiat. Quart., 78, 309-316, https://doi.org/10.1007/s11126007-9056-9, 2007.

Gee, J. P.: What Video Games Have to Teach Us About Learning and Literacy, Comput. Entertain., 1, 20-20, https://doi.org/10.1145/950566.950595, 2003.

Granic, I., Lobel, A. M., and Engels, R. C. M. E.: The benefits of playing video games, Am. Psychol., 69, 66-78, https://doi.org/10.1037/a0034857, 2014.

Griffiths, M. D.: The Educational Benefits of Videogames, Education and Health, 20, 47-51, 2002.

Hut, R.: Online Survey: Into the Wild: How Realistic Are Artistic Renderings?, available at: http://rolfhut.nl/botw (last access: 9 August 2019), 2018.

Kaplan, R. M. and Saccuzzo, D. P.: Psychological testing: Principles, applications, and issues, Nelson Education, Toronto, Canada, 2017.

Lord, F. M. and Novick, M. R.: Statistical theories of mental test scores, Information Age Publishing (IAP), Charlotte, NC, USA, 2008.

LunaPic: LunaPic.Com Photo Editor Van Gogh Tool, available at: https://www.lunapic.com/editor/?action=vangogh (last access: 9 August 2019), 2015.

Mayer, R. E.: Computer Games in Education, Annu. Rev. Psychol., 70, 531-549, 2019.

Mayo, M. J.: Video Games: A Route to Large-Scale STEM Education?, Science, 323, 79-82, 2009.

Mozelius, P., Fagerström, A., and Söderquist, M.: Motivating Factors and Tangential Learning for Knowledge Acquisition in Educational Games, Electronic Journal of e-Learning, 15, 343-354, 2017.

Newman, G., Wiggins, A., Crall, A., Graham, E., Newman, S., and Crowston, K.: The Future of Citizen Science: Emerging Technologies and Shifting Paradigms, Front. Ecol. Environ., 10, 298 304, https://doi.org/10.1890/110294, 2012.

Portnow, J.: Tangential Learning - How Games Can Teach Us While We Play - Extra Credits, YouTube, available at: https: //www.youtube.com/watch?v=rlQrTHrwyxQ\&t=7s (last access: 9 August 2019), 2012.

Reddit: R/Reddeadredemption - How Big EXACTLY Is the New Map? A Detailed Analysis, available at: https: //www.reddit.com/r/reddeadredemption/comments/9rgmbp/ how_big_exactly_is_the_new_map_a_detailed_analysis/ (last access: 9 August 2019), 2018.

Rodríguez Estrada, F. C. and Davis, L. S.: Improving Visual Communication of Science Through the Incorporation of Graphic Design Theories and Practices Into Science Communication, Sci. Commun., 37, 140-148, https://doi.org/10.1177/1075547014562914, 2015.

Squire, K.: Cultural Framing of Computer/Video Games, Game Studies, 2, 1-13, 2002. 
Squire, K.: Video Games in Education, Int. J. Intell. Games \& Simulation, 2, 49-62, 2003.

Wang, B., Taylor, L., and Sun, Q.: Families That Play Together Stay Together: Investigating Family Bonding through Video Games, New Media Soc., 20, 4074-4094, 2018.
Webster, A.: The Legend of Zelda: Breath of the Wild's Map Is Based on Kyoto, available at: https://www.theverge.com/2017/ 3/6/14827832/the-legend-of-zelda-breath-of-the-wild-mapkyoto-japan (last access: 9 August 2019), 2017. 